\title{
The Study of the Flat Grinding Process of Workpieces of Composite Materials
}

\author{
Valeriy Sapunov ${ }^{1, *}$, Nikolai Vetkasov $^{1}$, and Sergey Varlamov ${ }^{1}$ \\ ${ }^{1}$ Ulyanovsk State Technical University, Machine building faculty, 32 Severnyy Venets st, Ulyanovsk, \\ 432027, Russia
}

\begin{abstract}
The paper shows the results of the carried out examination of the grit of the processed surface and the intensiveness of the flat grinding of workpieces of composite materials. The processing has been carried out using of a special grinding tool with anti-frictional fillers produced in accordance with the microwave technology. The grit and components of grinding forces of workpieces of aluminomatrix composite materials with various carbon contents have been determined.
\end{abstract}

\section{Introduction}

Now that science and technology have caught on, requirements applied to structural materials become tougher. Specifically, new composite materials with lower density, able to retain their physical and mechanical properties at elevated temperatures, are sought-after in many industrial fields [1-4]. Heat-treated composite materials, resulting from aluminum carbon mechanical alloying, find ever-growing use in the aerospace field, machine engineering, energetics, medicine [5-9]. With the appearance of new materials the world faced with the issue to develop the best available technologies of their mechanical processing.

When flat grinding of metallocomposites, particularly carbon-alloyed aluminomatrix composite materials, additional requirements are specified for the equipment and tools related to providing of the minimum radial run-out of the working surface of the wheel and the minimum clogging level of the grinding tool. It is possible to achieve the desired result by using of the special grinding tool with anti-frictional fillers produced in accordance with the microwave technology providing the good health of the grinding tool [10-14]. This paper shows some results of the experimental study of the health of grinding wheels on a bakelite bunch with graphite fillers and bakelized in the microwave field when flat grinding of workpieces of carbon-alloyed aluminomatrix composite materials.

\section{Materials}

To obtain experimental specimens of the composite material, the following basic materials have been used: the primary dispersed aluminum powder PAD-1 (STO22436138-006-

\footnotetext{
* Corresponding author: sapunov_vv@mail.ru
} 
2006), the technical carbon powder K 354 (GOST 7885). The aluminum powder has been primarily passed through a $30-\mu$ screen. The amorphous carbon granulated powder has been originally grinded in the mechanical reactor (MR) during 10 minutes to destroy pellets and agglomerations. The alloying process has been carried out in MR. The carbon content in the mixture has been 3,4 and $5 \%$ by mass [15-17].

The obtained composite granule mixture has been cold-compacted under the pressure of $600 \mathrm{MPa}$ to the density $2,4 \mathrm{~g} / \mathrm{cm}^{3}$. Specimens have been heat-treated in the argon medium at exposure of 5 hours under the temperature $530{ }^{\circ} \mathrm{C}$. An X-ray phased and structural analysis has been carried out with the aid of the diffractometer DRON-3M in Cu-radiation. The coherent scattering area (CSA) dimensions have been evaluated by diffraction line broadening. The CSA dimension has been identified with the average crystallite dimension D. In this case, the CSA dimension is somewhat lower than the same value obtained with the help of electronic microscopy, since the CSA reflects the inner (ordered) crystallite area which excludes highly distorted boundaries [18-20]. Formulation and concentration growth of aluminum carbide hardening dispersoids have been assessed by formation and densification of diffraction lines. The microhardness has been measured with the help of the durometer type Tukon 1102 Micro Vickers (Vickers technique) under the indentor load 100 $\mathrm{g}$ and the exposure time $10 \mathrm{~s}$ (Table 1).

Table 1. Parameters of CM specimens for tests.

\begin{tabular}{|c|c|c|c|}
\hline Designation & Carbon content, $\%$ & Microhardness, MPa & $\begin{array}{c}\text { Crystallite dimension, } \\
\mathrm{nm}\end{array}$ \\
\hline AMK-3 & 3 & 65 & 51 \\
\hline AMK-4 & 4 & 89 & 44 \\
\hline AMK-5 & 5 & 103 & 40 \\
\hline
\end{tabular}

\section{Research technique}

Comparative technological tests have been carried out using of the experimental installation mounted on the basis of the flat grinding machine (mod. 3E711BФ2). The automated working station has been created for measuring of force components and the average contact temperature. This automated working station gives an opportunity to tune settings of test scenarios, to store and to search for a relevant scenario in the database, to carry out the cut-through calibration of measuring channels, a real-time measuring with simultaneous archiving and visualizing of test data, to look through and to analyze the results. Grinding wheels (GW) on a bakelite bunch 1-300'40'76' 14A50C15Б with a graphite filler $4 \%$ (of weight) have been used as grinding tools. Their semi-finished products have been heattreated (bakelized) in the microwave field using of the original experimental and industrial microwave installation.

The hardness of GW has been controlled by the acoustic method using of the device «Zvuk-203M». The mechanical performance of wheels has been tested at the stand «SIP800». Tests have been carried out on prismatic specimens with the length of $45 \mathrm{~mm}$ and the section of $15 \times 15$. Specimens have been produced of the one batch of materials with the hardness diffusion not exceeding of $\pm 4 \%$. Before beginning of each series of tests the batch of specimens have been grinded to one and the same size with the $7^{\text {th }}$ tolerance grade and the grit $\mathrm{Ra} \leq 1,6 \mu \mathrm{m}$. 


\section{Study results}

After finishing of the calibration of the dynamometer, grit tests of the processed surface and intensiveness tests of the flat grinding of workpieces of aluminomatrix composite materials with various carbon content, produced using of the mechanical alloying method, have been carried out. Test results are shown in the Table 2 and the Table 3.

Table 2. Values of force components when grinding of different marks of composite materials.

\begin{tabular}{|c|c|c|c|c|c|c|}
\hline \multirow{3}{*}{$\begin{array}{c}\text { № of } \\
\text { test }\end{array}$} & \multicolumn{5}{|c|}{ Designation of the composite material } \\
\cline { 2 - 7 } & \multicolumn{7}{|c|}{ AMK-3 } & \multicolumn{2}{c|}{ AMK-4 } & \multicolumn{2}{c|}{ AMK-5 } \\
\cline { 2 - 7 } & $P_{y}$ & $P_{z}$ & $P_{y}$ & $P_{z}$ & $P_{y}$ & $P_{z}$ \\
\cline { 2 - 7 } & 11,8 & 8,2 & 13,4 & 9,6 & 14,9 & 10,8 \\
\hline 1 & 12,4 & 8,5 & 13,9 & 9,3 & 14,7 & 11,1 \\
\hline 2 & 11,5 & 8,4 & 13,1 & 9,9 & 15,5 & 10,7 \\
\hline 3 & & &
\end{tabular}

Table 3. Values of the arithmetical mean deviation of the profile Ra of specimens of composite materials after the flat grinding operation.

\begin{tabular}{|c|c|c|c|}
\hline \multirow{2}{*}{ № of test } & \multicolumn{3}{|c|}{ Designation of the composite material } \\
\cline { 2 - 4 } & AMK-3 & AMK-4 & AMK-5 \\
\hline 1 & 1,34 & 1,1 & 0,9 \\
\hline 2 & 1,39 & 1,21 & 0,84 \\
\hline 3 & 1,31 & 1,08 & 0,83 \\
\hline
\end{tabular}

\section{Conclusions}

The carried out tests justified the application efficiency of the special grinding tool with anti-frictional fillers produced in accordance with the microwave technology when processing of carbon-alloyed aluminomatrix composite materials. Application of the special grinding tool has given an opportunity to extend by 1,8 times the health of the tool and to reduce the grit of the processed surface. It has been made clear that increasing of the carbon content in the processed specimens leads to the increase in grinding forces. The longest health of the tool has been noted when processing of specimens with the maximum carbon content. The grit of the processed surface is slightly reduced with the increase in the carbon content in the processed specimens. This can be explained with the increased microhardness of specimens and the decreased health of the wheel.

\section{References}

1. S.S. Narayanan, G. Manickavasaham, S. Moorthy, Experimental Investigation of Aluminium alloy with MWCNT Composite to increase the echanical Properties by Stir Casting Method, IOSR Journal of Mechanical and Civil Engineering, v. 12 (4) ver. II, pp. 30-34 (2015) 
2. E.P. Shalunov, M.A. Shvedov, I.V. Arkhipov, Synthesis of dispersoids in the reaction mechanical alloying of powdered aluminum with carbon, Bulletin of the Chuvash University, 2 pp. 165-172 (2014)

3. C. Yang, Y. Zong, Z. Zheng, D. Shan, Experimental and theoretical investigation on the compressive behavior of aluminum borate whisker reinforced 2024Al composites, Materials Characterization, 96, pp. 84-92 (2014)

4. L. Wang, H. Choi, J.M. Myoung, W. Lee, Mechanical alloying of multi-walled carbon nanotubes and aluminium powders for the preparation of carbon/metal composites, Carbon, 47, pp. 3427-3433 (2009)

5. M. Susniak, J. Karwan-Baczewska, J. Dutkiewicz, M. Actis Grande, M. Rosso, An experimental study of aluminum alloy matrix composite reinforced sic made by hot pressing method, Archives of Metallurgy and Materials, 60, pp. 1523-1527 (2015)

6. Yu.V. Kuzmich, I.G. Kolesnikova, V.I. Serba, B.M. Freudin, Mechanical alloying (Science, Moscow, 2005)

7. S.J. Yoo, S.H. Han, W.J. Kim, Strength and strain hardening of aluminum matrix composites with randomly dispersed nanometer-length fragmented carbon nanotubes, Scr. Mater., 68, pp. 711-714 (2013)

8. L. Ci, Z. Ryu, P.N.Y. Jin, M. Rühle, Investigation of the interfacial reaction between multi-walled carbon nanotubes and aluminum, Acta. Mater., 54, pp. 5367-5375 (2006)

9. N. Nayan, S.V.S.N. Murty, S.C. Sharma, K.S. Kumar, P.P. Sinha, Calorimetric study on mechanically milled aluminum and multiwall carbon nanotube composites, Materials Characterization, 62, pp. 1087-1093 (2011)

10. V. Sapunov, N. Vetkasov, Simulation of the temperature distribution in semi-finished products of combined abrasive tools during their microwave heating, IOP Conference Series: Materials Science and Engineering, 709, 044055 (2020)

11. R. Komanduri, Z.B. Hou, International journal of Mechanical Sciences, 43-1, 57-88 (2001).

12. Yu.A. Sizyy, M.S. Stepanov Eastern-European Journal of Enterprise Technologies, 2-8, 52-63 (2004).

13. N. Vetkasov, S. Ulitin, O. Krupennikov, V. Sapunov, A. Ankilov, Simulation of thermal strength of the process of flat grinding of workpieces in intermittent circles, MATEC Web of Conferences, 298, 00022 (2019)

14. V. Sapunov, N. Vetkasov, L. Khudobin, The study of the health of grinding wheels on a bakelite bunch, heat-treated in a microwave field, Materials Today: Proceedings, v. 38(4), pp. 1711-1713 (2021)

15. N. Vetkasov, A. Kapustin, V. Sapunov, Development and Investigation of The Production Process of the Aluminum Matrix Composition Mechanically Alloyed With Single-Walled Carbon Nanotubes and the Composition Material Made of it, MATEC Web of Conferences, 297, 04001 (2019)

16. N. Vetkasov, A. Kapustin,V. Sapunov, Study of the process of mechanical doping of aluminum with nanoscale allotropic carbon modifications, Materials Today: Proceedings, v. 19(5), pp. 2299-2303 (2019)

17. N. Vetkasov, L. Khudobin,V. Sapunov, S. Varlamov, Obtaining, microstructure and microhardness of carbon-doped aluminomatrix composites, Materials Today: Proceedings, v. 38(4), pp. 1706-1710 (2021)

18. D.Yu. Pushcharovsky, X-ray analysis of minerals (Geoinformmak, Moscow, 2000).

19. A.I. Gusev, Nanomaterials, nanostructures, nanotechnologies, second edition (Science - Fizmatlit, Moscow, 2007)

20. A.I. Gusev, A.S. Kurlov, Certification of nanocrystalline materials by particle size (grains), Metallophysics and the latest technology, 30, pp. 679-694 (2008) 